\title{
TOTAL CHARACTERS AND CHEBYSHEV POLYNOMIALS
}

\author{
EIRINI POIMENIDOU and HOMER WOLFE
}

Received 8 January 2002

\begin{abstract}
The total character $\tau$ of a finite group $G$ is defined as the sum of all the irreducible characters of G. K. W. Johnson asks when it is possible to express $\tau$ as a polynomial with integer coefficients in a single irreducible character. In this paper, we give a complete answer to Johnson's question for all finite dihedral groups. In particular, we show that, when such a polynomial exists, it is unique and it is the sum of certain Chebyshev polynomials of the first kind in any faithful irreducible character of the dihedral group $G$.
\end{abstract}

2000 Mathematics Subject Classification: 20C15.

1. Introduction. A Gel'fand model $M$ for a group $G$ was defined in [8] as any complex representation of $G$ which is isomorphic to the direct sum of all irreducible representations of $G$. We refer to the character of such a representation as the total character $\tau$ of $G$. A Gel'fand model afforded by a (generalized) permutation representation is referred to as a (weakly) geometric Gel'fand model. When a Gel'fand model $M$ is the representation afforded by a nonnegative integer linear combination of powers of a genuine $G$-set $X$, then the total character $\tau$ of $G$ can be expressed as a polynomial in the character $\chi$ afforded by $X$. A question related to this idea was posed by Johnson [5].

QUESTION 1.1. For a finite group $G$, do there necessarily exist an irreducible character $\chi$ and a monic polynomial $f(x) \in \mathbb{Z}[x]$ such that $f(x)=\tau$, where $\tau$ is the total character of $G$ ?

Johnson's question arose in the context of character sharpness which we will briefly explain. Let $G$ be a finite group, $\chi$ a generalized character of $G$ of degree $n, L=\{\chi(g) \mid g \neq 1\}$, and $f_{L}(x)=\prod_{l \in L}(x-l)$. It was discovered by Blichfeldt [2] and rediscovered in a modern context by Kiyota [6] that $f_{L}(x) \in \mathbb{Z}[x]$ and $|G|$ divides $f_{L}(n)$. In the special case that $f_{L}(n)=|G|$, the character $\chi$ is said to be sharp. Another way to characterize a sharp character is to notice that the class function $f_{L}(\chi)=\prod_{l \in L}\left(\chi-l 1_{G}\right)=\rho$, where $\rho$ is the regular character and $1_{G}$ is the trivial character of $G$. In other words, every irreducible character of $G$ appears as a constituent of $f_{L}(\chi)$.

A partial answer to Johnson's question was given in [7] for certain dihedral groups. In this paper, we give a complete treatment for all dihedral groups and we show that the right polynomials, when they exist, are integer sums of Chebyshev polynomials of the first kind. 
We prove the following main theorem.

THEOREM 1.2. Let $G \cong D_{2 n}$ and let $\mathrm{T}$ be the total character of $G$.

(1) If $n$ is odd or if $n=2 m$, where $m \equiv 2,3(\bmod 4)$, then there exists a unique monic polynomial $P(x) \in \mathbb{Z}[x]$ such that $P(X)=\tau$ for any faithful $x \in \operatorname{Irr}(G)$. Moreover,

$$
P(x)= \begin{cases}2 \sum_{k=0}^{m} T_{k}\left(\frac{x}{2}\right)-2 T_{m-1}\left(\frac{x}{2}\right), & \text { if } n=2 m-1, n \equiv 1,3(\bmod 8), \\ 2 \sum_{k=0}^{m-1} T_{k}\left(\frac{x}{2}\right), & \text { if } n=2 m-1, n \equiv 5,7(\bmod 8), \\ 2 \sum_{k=0}^{m} T_{k}\left(\frac{x}{2}\right), & \text { if } n=2 m, m \equiv 2,3(\bmod 4),\end{cases}
$$

where $T_{k}(x)$ is the kth Chebyshev polynomial of the first kind.

(2) If $n=2 m$ and $m \equiv 1(\bmod 4)$, then there exists a unique monic polynomial $P(x) \in \mathbb{Z}[x]$ such that $P(\chi)=2 \tau$ for any faithful $\chi \in \operatorname{Irr}(G)$, where

$$
P(x)=2\left[T_{m+1}\left(\frac{x}{2}\right)+2 \sum_{k=0}^{m} T_{k}\left(\frac{x}{2}\right)-T_{m-1}\left(\frac{x}{2}\right)\right] .
$$

Moreover, there does not exist $P(x) \in \mathbb{Z}[x]$ such that $P(x)=\tau$ for any $\chi \in \operatorname{Irr}(G)$.

(3) If $G \cong D_{2 n}$, where $n \equiv 0(\bmod 8)$, then there does not exist a polynomial $P(x) \in \mathbb{C}[x]$ such that $P(\chi)=\tau$ for any $\chi \in \operatorname{Irr}(G)$.

2. Preliminaries. The total character for all dihedral groups was computed in [7, Proposition 2.1]. A dihedral group of order $2 n$ with $n \geq 3$ will be presented as usual as

$$
D_{2 n}=G=\left\langle a, b: a^{n}=b^{2}=1, b^{-1} a b=a^{-1}\right\rangle .
$$

Using the notation in [4], we use $g_{i}$ and $h_{i}$ to denote a representative and the size of the $i$ th conjugacy class, respectively. The character table and total character $\tau$ of $D_{2 n}$ are given below.

CASE 1 ( $n$ odd). The conjugacy classes of $D_{2 n}(n$ odd $)$ are

$$
\{1\}, \quad\left\{a^{r}, a^{-r}\right\} \quad\left(1 \leq r \leq \frac{n-1}{2}\right), \quad\left\{a^{s} b \mid 0 \leq s \leq n-1\right\} .
$$

The character table of $D_{2 n}(n$ odd $)$ and the total character $\tau$, where $\epsilon=e^{2 \pi i / n}$, is presented in Table 2.1. 
TABLE 2.1

\begin{tabular}{c|ccc}
\hline$g_{i}$ & 1 & $a^{r}(1 \leq r \leq(n-1) / 2)$ & $b$ \\
$h_{i}$ & 1 & 2 & $n$ \\
\hline$\chi_{1}$ & 1 & 1 & 1 \\
$\chi_{2}$ & 1 & 1 & -1 \\
$\psi_{j}(1 \leq j \leq(n-1) / 2)$ & 2 & $\epsilon^{j r}+\epsilon^{-j r}$ & 0 \\
\hline$\tau$ & $n+1$ & 1 & 0 \\
\hline
\end{tabular}

CASE 2 ( $n$ even). If $n$ is even, say $n=2 m$, then the conjugacy classes of $D_{2 n}$ are

$$
\begin{aligned}
\{1\}, \quad & \left\{a^{m}\right\}, \quad\left\{a^{r}, a^{-r}\right\} \quad(1 \leq r \leq m-1), \\
& \left\{a^{s} b \mid s \text { even }\right\}, \quad\left\{a^{s} b \mid s \text { odd }\right\} .
\end{aligned}
$$

The character table of $D_{2 n}$ ( $n$ even, $n=2 m$, and the total character $\tau$, where $\epsilon=e^{2 \pi i / n}$ ) is given in Table 2.2.

TABLE 2.2

\begin{tabular}{c|ccccc}
\hline$g_{i}$ & 1 & $a^{m}$ & $a^{r}(1 \leq r \leq m-1)$ & $b$ & $a b$ \\
$h_{i}$ & 1 & 1 & 2 & $n / 2$ & $n / 2$ \\
\hline$\chi_{1}$ & 1 & 1 & 1 & 1 & 1 \\
$\chi_{2}$ & 1 & 1 & 1 & -1 & -1 \\
$\chi_{3}$ & 1 & $(-1)^{m}$ & $(-1)^{r}$ & 1 & -1 \\
$\chi_{3}$ & 1 & $(-1)^{m}$ & $(-1)^{r}$ & -1 & 1 \\
$\psi_{j}(1 \leq j \leq m-1)$ & 2 & $2(-1)^{j}$ & $\epsilon^{j r}+\epsilon^{-j r}$ & 0 & 0 \\
\hline & $2(m+1)$ & $0, \quad m$ odd, & $0, \quad r$ odd, & 0 & 0 \\
$\tau$ & & $2, \quad m$ even, & $2, \quad r$ even, & & \\
\hline
\end{tabular}

The $n$th Chebyshev polynomial of the first kind is defined as $T_{n}(x)=$ $\cos \left(n \cos ^{-1}(x)\right)$ for $|x| \leq 1$. Chebychev polynomials can be expressed recursively as $T_{n+2}(x)=2 x T_{n+1}(x)-T_{n}(x), T_{0}(x)=1$, and $T_{1}(x)=x$. Before we proceed with the proof of Theorem 1.2 we need the following lemmas.

LEMMA 2.1. If $P\left(\psi_{1}\right)=\tau$ for some $P(x) \in \mathbb{Z}[x]$, then $P\left(\psi_{j}\right)=\tau$ for any faithful character $\psi_{j}$ of $D_{2 n}$.

Proof. We first observe that $\psi_{j}$ is faithful if and only if $(j, n)=1$, for $\epsilon^{j r}+\epsilon^{-j r}=2 \cos (2 \pi r j / n)=2$ if and only if $n \mid r j$ if and only if $(n, j) \neq 1$. 
Hence for $(j, n)=1$, the character $\psi_{j}$ is faithful and a Galois conjugate of $\psi_{1}$ with the same set of character values. Since for $n$ odd $P\left(\psi_{1}\left[a^{r}\right]\right)=1$ for all $r$, it follows that $P\left(\psi_{j}\left[a^{r}\right]\right)=P\left(\psi_{1}\left[a^{r}\right]\right)=1$. When $n$ is even, we have that $P\left(\psi_{1}\left[a^{r}\right]\right)=2$ when $r$ is even and $P\left(\psi_{1}\left[a^{r}\right]\right)=0$ when $r$ is odd. For a faithful $\psi_{j}$, the set of character values is the same as those of $\psi_{1}$; and since $j$ is necessarily odd, it follows that $P\left(\psi_{j}\left[a^{r}\right]\right)=2$ when $r$ is even, and $P\left(\psi_{j}\left[a^{r}\right]\right)=$ 0 when $r$ is odd as required.

LEMMA 2.2. Let the $n$th Chebyshev polynomial of the first kind be expressed as $T_{n}(x)=\sum_{k=1}^{n} c_{n, k} x^{k}+c_{n, 0}$. Then $c_{n, n}=2^{(n-1)}$ and $2^{(k-1)} \mid c_{n, k}$ for $1 \leq k \leq n$.

Proof. The first half follows easily by an inductive argument on $n$ and by using the recursive relation mentioned above. For divisibility of the coefficients, observe that the result is true for $n=1$ and $n=2$. Now assume that the result is true for all Chebyshev polynomials of degree less that $n$ and consider the $n$th degree Chebyshev polynomial $T_{n}(x)$. We have by the recursive relation that $c_{n, k}=2 c_{n-1, k-1}-c_{n-2, k}$. By inductive hypothesis, $2^{(k-1)} \mid 2 c_{n-1, k-1}-c_{n-2, k}$ for any $1 \leq k \leq n$. Hence the result is true for all the coefficients of $T_{n}(x)$ and hence for all $n$.

LEMMA $2.3[3,134.2]$. For $m \in \mathbb{Z}^{+}$,

$$
\sum_{k=0}^{m} \cos k x=\frac{1}{2}\left[1+\frac{\sin ((m+1 / 2) x)}{\sin ((1 / 2) x)}\right] .
$$

LEMMA 2.4. Let $p_{m}(x)=2 \sum_{k=0}^{m} T_{k}(x / 2)$. The following equations hold:

$$
\begin{aligned}
& p_{m}(2)=2(m+1) ; \\
& p_{m}\left(2 \cos \frac{2 \pi r}{2 m}\right)= \begin{cases}0, & \text { if } r \text { odd }, \\
2, & \text { if } r \text { even; }\end{cases} \\
& p_{m}\left(2 \cos \left(\frac{2 \pi r}{(2 m-1)}\right)\right)=1+(-1)^{r} 2 \cos \left(\frac{\pi r}{(2 m-1)}\right) ; \\
& p_{m}(0)=1+\sin \left(\frac{m \pi}{2}\right)+\cos \left(\frac{m \pi}{2}\right) .
\end{aligned}
$$

Proof. Equation (2.5) follows immediately since $T_{k}(1)=1$, for all $k \geq 0$. For the other equations, we use Lemma 2.3,

$$
\begin{aligned}
p_{m}\left(2 \cos \left(\frac{2 \pi r}{2 m}\right)\right) & =2 \sum_{k=0}^{m} \cos \left(\frac{\pi k r}{m}\right) \\
& =1+\frac{\sin ((m+1 / 2)(\pi r / m))}{\sin (\pi r / 2 m)} \\
& =1+\cos (\pi r)= \begin{cases}0, & \text { if } r \text { odd } \\
2, & \text { if } r \text { even, }\end{cases}
\end{aligned}
$$




$$
\begin{aligned}
p_{m}\left(2 \cos \left(\frac{2 \pi r}{2 m-1}\right)\right) & =1+\frac{\sin (((2 m-1)+2) / 2)(2 \pi r /(2 m-1))}{\sin (\pi r /(2 m-1))} \\
& =1+\cos (\pi r) \frac{\sin 2(\pi r /(2 m-1))}{\sin (\pi r /(2 m-1))} \\
& =1+(-1)^{r} 2 \cos \left(\frac{\pi r}{2 m-1}\right), \\
p_{m}(0) & =2 \sum_{k=0}^{m} T_{k}(0)=2 \sum_{k=0}^{m} \cos \left(\frac{k \pi}{2}\right) \\
& =1+\frac{\sin ((m+1 / 2)(\pi / 2))}{\sin (\pi / 4)} \\
& =1+\sin \left(\frac{m \pi}{2}\right)+\cos \left(\frac{m \pi}{2}\right) .
\end{aligned}
$$

3. Proof of main theorem. It is a well-known theorem of polynomial interpolation that there exists a unique polynomial of degree $k$ or less that maps $k+1$ distinct points in the domain to predefined points in the range. We refer the reader to [1] for a natural proof. Thus, by presenting a polynomial of degree less than the number of distinct values of some $\chi \in \operatorname{Irr}(G)$, which matches the values of the total character when considered as a class function on $\chi$, we immediately get that the polynomial is both minimal and unique.

Proof OF TheOrem 1.2(1). Let $n=2 m-1, n \equiv 1,3(\bmod 8)$. Using Lemma 2.4 , we have that $P(x)=p_{m}(x)-2 T_{m-1}(x / 2)$,

$$
\begin{aligned}
P\left(\psi_{1}[1]\right) & =2(m+1)-2=2 m=n+1=\tau[1], \\
P\left(\psi_{1}\left[a^{r}\right]\right) & =1+(-1)^{r} 2 \cos \left(\frac{\pi r}{2 m-1}\right)-2 \cos \left(\frac{\pi r(m-1)}{2 m-1}\right) \\
& =1=\tau\left[a^{r}\right], \\
P\left(\psi_{1}[b]\right) & =1+\sin \left(\frac{m \pi}{2}\right)+\cos \left(\frac{m \pi}{2}\right)-2=0=\tau[b] .
\end{aligned}
$$

Let $n=2 m-1, n \equiv 5,7(\bmod 8)$. Then $P(x)=p_{m-1}(x)$,

$$
\begin{aligned}
P\left(\psi_{1}[1]\right) & =(2(m-1)+1)=2 m=n+1=\tau[1], \\
P\left(\psi_{1}\left[a^{r}\right]\right) & =2 \sum_{k=0}^{m-1} \cos \left(\frac{2 \pi k r}{2 m-1}\right) \\
& =1+\frac{\sin (\pi r)}{\sin (\pi r /(2 m-1))}=1=\tau\left[a^{r}\right], \\
P\left(\psi_{1}[b]\right) & =1+\sin \left(\frac{m \pi}{2}\right)+\cos \left(\frac{m \pi}{2}\right)=0=\tau[b] .
\end{aligned}
$$


Let $n=2 m, m \equiv 2,3(\bmod 4)$. Then $P(x)=p_{m}(x)$,

$$
\begin{aligned}
P\left(\psi_{1}[1]\right) & =2(m+1)=\tau[1], \\
P\left(\psi_{1}\left[a^{r}\right]\right) & = \begin{cases}0, & \text { if } r \text { odd }, \\
2, & \text { if } r \text { even, }\end{cases} \\
& =\tau\left[a^{r}\right], \\
P\left(\psi_{1}[b]\right) & =P\left(\psi_{1}[a b]\right)=1+\sin \left(\frac{m \pi}{2}\right)+\cos \left(\frac{m \pi}{2}\right)=0 \\
& =\tau[b]=\tau[a b] .
\end{aligned}
$$

Thus, in each of the above three cases, $P(x)$ is minimal, and by Lemma 2.2 it is monic with integer entries. By Lemma 2.1, we have that $P\left(\psi_{j}\right)=\tau$ for any faithful $\psi_{j} \in \operatorname{Irr}(G)$.

Proof of TheOrem 1.2(2). Let $n=2 m$ and $m \equiv 1(\bmod 4)$. Then $P(x)=$ $2 T_{m+1}(x / 2)+2 p_{m}(x)-2 T_{m-1}(x / 2)$,

$$
\begin{aligned}
P\left(\psi_{1}[1]\right) & =2[1+2(m+1)-1]=2 \tau[1], \\
P\left(\psi_{1}\left[a^{r}\right]\right) & =2\left[\cos \frac{(m+1) \pi r}{m}+1+(-1)^{r}-\cos \frac{(m-1) \pi r}{m}\right] \\
& = \begin{cases}0, & \text { if } r \text { odd }, \\
4, & \text { if } r \text { even, }\end{cases} \\
& =2 \tau\left[a^{r}\right], \\
P\left(\psi_{1}[b]\right) & =P\left(\psi_{1}[a b]\right) \\
& =2\left[0+\left(1+\sin \left(\frac{m \pi}{2}\right)+\cos \left(\frac{m \pi}{2}\right)\right)-0\right] \\
& =0=2 \tau[b]=2 \tau[a b] .
\end{aligned}
$$

Again, we have that $P(x)$ is minimal since it matches $m+2$ distinct values with a polynomial of degree $m+1$. By Lemma 2.1, $P\left(\psi_{j}\right)=2 \tau$ for any faithful character of $G$, and by Lemma 2.2 it is monic with integer entries. Thus, the unique polynomial that maps the values of a faithful $\chi \in \operatorname{Irr}(G)$ onto $\tau$ is $(1 / 2) P(x)$ and has noninteger coefficients.

Proof OF TheOREM 1.2(3). Let $n=2 m$ and $m \equiv 0(\bmod 4)$. When $r=m / 2$, we have that for any faithful character, $\psi_{j}\left[a^{m / 2}\right]=\psi_{j}(b)=0$, but $\tau\left[a^{m / 2}\right]=2$ and $\boldsymbol{\tau}(\boldsymbol{b})=0$; thus we have an inconsistent system.

ACKNOWLEDgments. Both authors wish to thank Professor Donald Colladay of New College and Professor Kenneth Johnson of Pennsylvania State University for their comments and suggestions during writing this paper. We 
also wish to thank the New College Foundation for supporting this project through a faculty/student development grant.

\section{REFERENCES}

[1] Ȧ. Björck and G. Dahlquist, Numerical Methods, Prentice-Hall Series in Automatic Computation, Prentice-Hall, New Jersey, 1974.

[2] H. F. Blichfeldt, A theorem concerning the invariants of linear homogeneous groups, with some applications to substitution-groups, Trans. Amer. Math. Soc. 5 (1904), no. 4, 461-466.

[3] I. S. Gradshteyn and I. M. Ryzhik, Table of Integrals, Series, and Products, Academic Press, Massachusetts, 1994.

[4] G. James and M. Liebeck, Representations and Characters of Groups, Cambridge Mathematical Textbooks, Cambridge University Press, Cambridge, 1993.

[5] K. W. Johnson, private correspondence, 1997.

[6] M. Kiyota, An inequality for finite permutation groups, J. Combin. Theory Ser. A 27 (1979), no. 1, 119.

[7] E. Poimenidou and A. Cottrell, Total characters of dihedral groups and sharpness, Missouri J. Math. Sci. 12 (2000), no. 1, 12-25.

[8] J. Soto-Andrade, Geometrical Gel'fand models, tensor quotients, and Weil representations, The Arcata Conference on Representations of Finite Groups (Arcata, Calif., 1986), Proc. Sympos. Pure Math., vol. 47, American Mathematical Society, Rhode Island, 1987, pp. 305-316.

Eirini Poimenidou: Division of Natural Sciences, New College of Florida, 5700 North Tamiami Trail, Sarasota, FL 34243, USA

E-mail address: poimenidou@ncf.edu

Homer Wolfe: Division of Natural Sciences, New College of Florida, 5700 North Tamiami Trail, Sarasota, FL 34243, USA

E-mail address: homer.wolfeancf.edu 


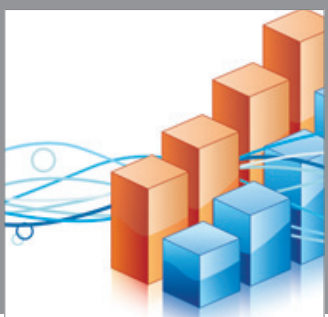

Advances in

Operations Research

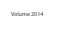

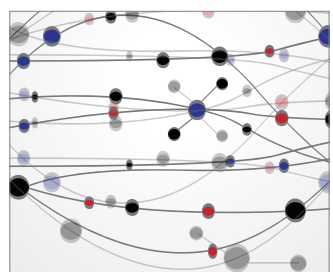

\section{The Scientific} World Journal
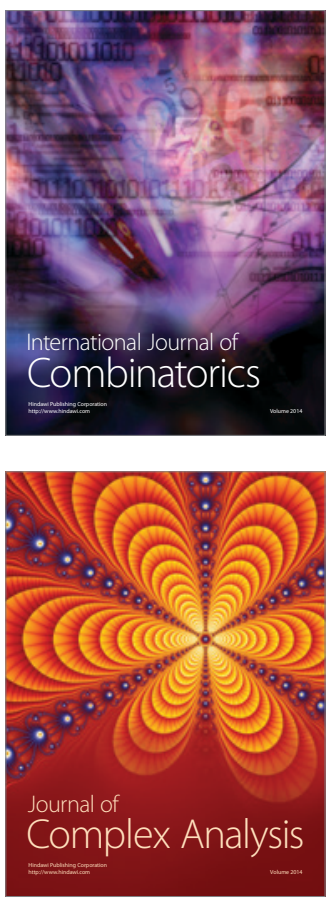

International Journal of

Mathematics and

Mathematical

Sciences
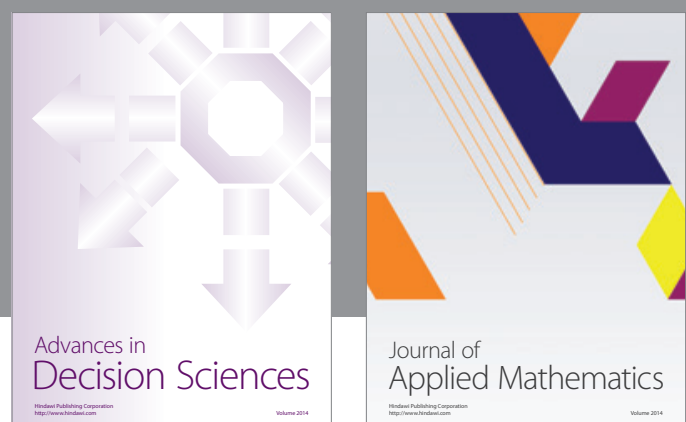

Journal of

Applied Mathematics
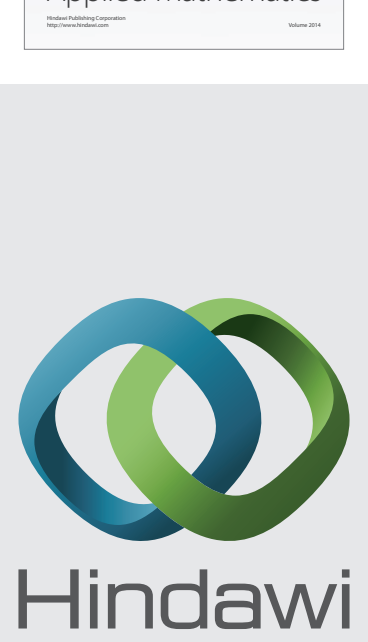

Submit your manuscripts at http://www.hindawi.com
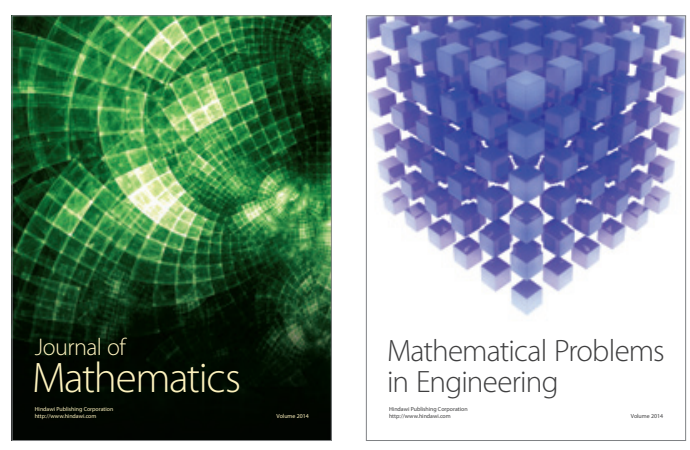

Mathematical Problems in Engineering
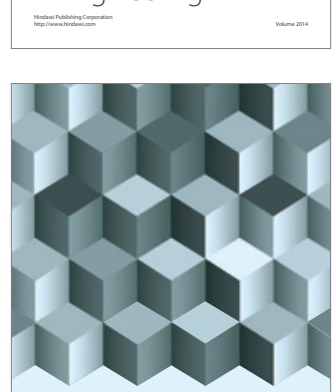

Journal of

Function Spaces
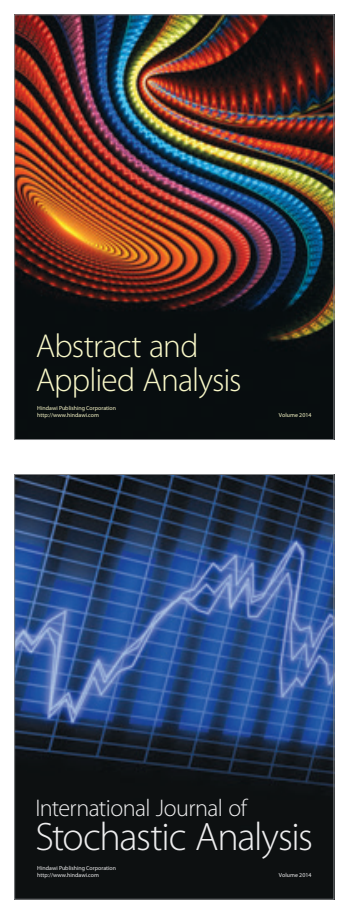

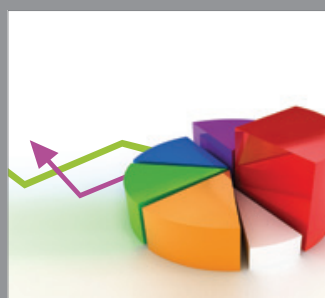

ournal of

Probability and Statistics

Promensencen
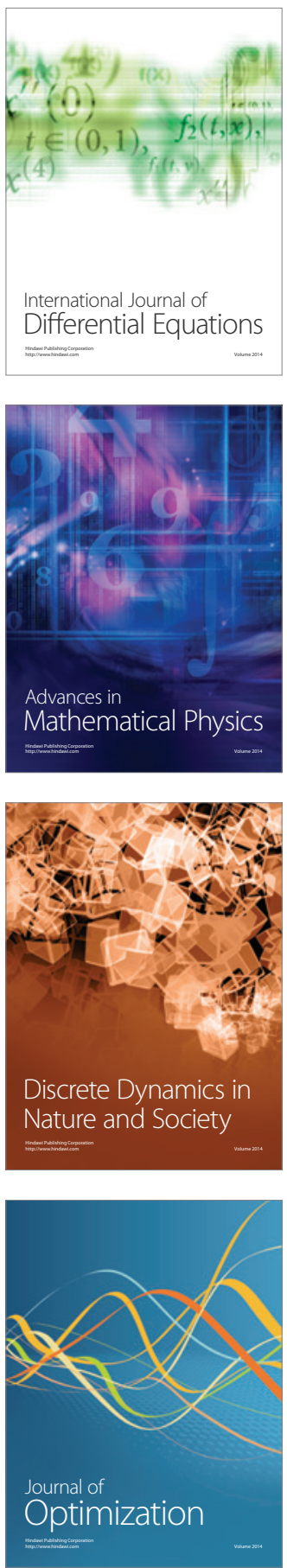